\title{
Seria o Ciúme Mediador entre as Experiências na Família de Origem e a Violência Física na Conjugalidade?
}

\author{
Karla Rafaela Haack ${ }^{1}$ \\ Denise Falcke ${ }^{1}$ \\ ${ }^{1}$ Universidade do Vale do Rio dos Sinos (Unisinos), São Leopoldo, Rio Grande do Sul, Brasil
}

\begin{abstract}
Resumo
A violência conjugal é um fenômeno multideterminado, o qual sofre impacto de experiências precoces e atuais. O objetivo deste estudo foi testar um modelo teórico no qual o ciúme é mediador entre as experiências na família de origem e a violência física conjugal. Trata-se de um estudo com delineamento quantitativo, transversal e explicativo. Contou com uma amostra de 600 pessoas, de 18 a 65 anos, residentes em 13 estados brasileiros. Os instrumentos foram questionário sociodemográfico, FBQ, CTS2 e ICR, em uma coleta por formulário eletrônico na internet. Modelagem de equações estruturais foi utilizada para testar a validade empírica do modelo teórico. Os resultados indicaram que o ciúme media parcialmente as experiências na família de origem e a ocorrência da violência conjugal. Os dados apontam para a necessidade de atenção ao ciúme nos relacionamentos amorosos, como forma de atuar na prevenção às situações de violência conjugal.

Palavras-chave: ciúme, família de origem, conflito conjugal, equações estruturais, conjugalidade
\end{abstract}

Is jealousy a mediator between experiences in the family of origin and physical violence in conjugality?

\begin{abstract}
Conjugal violence is a multidetermined phenomenon, which is impacted by early and present experiences. The objective of this study was to test a theoretical model in which jealousy mediates between experiences in the family of origin and physical domestic violence. This was a quantitative, transversal, and explanatory study, which counted on a sample of 600 people, aged from 18 to 65 years, from 13 Brazilian states. The instruments included a sociodemographic questionnaire, the FBQ, the CTS2, and the RJI, all collected electronically. Structural equation modeling was used to test the empirical validity of the theoretical model. The results indicated that jealousy partially mediates the experiences in the family of origin and the occurrence of conjugal violence. The data point to the need for attention to jealousy in love relationships, as a way of acting in the prevention of situations of conjugal violence.

Keywords: jealousy; family of origin; marital conflict; structural equations; conjugality.
\end{abstract}

Serián los celos mediadores entre las vivencias en la familia de origen y la violencia física en la conjugalidad?

\begin{abstract}
Resumen
La violencia conyugal es un fenómeno multideterminado, el cual sufre impacto de experiencias tempranas y actuales. El objetivo de este estudio fue testar un modelo teórico en el cual los celos son mediadores entre las vivencias en la familia de origen y la violencia física conyugal. Se trata de un estudio con delineamiento cuantitativo, transversal y explicativo. Se contó con una muestra de 600 personas, de 18 a 65 años, residentes en 13 estados brasileños. Los instrumentos fueron el cuestionario sociodemográfico, FBQ, CTS2 e ICR, recogidos por medio de formulario electrónico en internet. El modelo de ecuaciones estructurales fue utilizado para testar la validez empírica del modelo teórico. Los resultados indicaron que los celos midieron parcialmente las vivencias en la familia de origen y la ocurrencia de la violencia conyugal. Los datos señalan la necesidad de atención de los celos en las relaciones amorosas, como forma de actuar en la prevención de las situaciones de violencia conyugal.

Palabras clave: celos; familia de origen; conflicto conyugal; ecuaciones estructurales; conyugalidad
\end{abstract}

A violência, em todas as suas distintas manifestações, caracteriza-se por um problema de grande magnitude para a saúde pública, pelo impacto negativo na qualidade de vida dos envolvidos e dos cuidados de saúde necessários (Barreto, Teston, Latorre, Mathias, \& Marcon, 2016). Estudos nacionais indicam prevalência de violência física conjugal entre $5 \%$ e $25 \%$ (Colossi, Razera, Haack, \& Falcke, 2015; Madalena, Falcke, \& Carvalho, 2015).

A violência física pode ser entendida como atos violentos, uso de força física de forma intencional, não acidental, com o objetivo de ferir, lesar, provocar dor e sofrimento ou destruir a pessoa, deixando, ou não, marcas evidentes no seu corpo. Pode se manifestar por meio de tapas, beliscões, chutes, empurrões, queimaduras, dentre outras (OMS, 2002). Em muitas circunstâncias, causa lesão corporal, levando à necessidade de busca por auxílio médico após uma briga ou ocorrência de desmaio e quebra de algum osso e inclusive a ocorrência de homicídio. Pode ocorrer uma única vez, ou repetidas vezes e, quando não denunciada, existe chance de ocorrer uma escalada 
de intensidade e frequência (Hirigoyen, 2006). Destaca-se que a ocorrência de violência física pode ser concomitante com outros tipos (Panuzio \& DiLillo, 2010; Schraiber et al., 2007).

$\mathrm{Na}$ tentativa de compreender o fenômeno da violência, diversos estudos apontam que existe relação entre experiências na família de origem e a violência conjugal (Colossi, Marasca, \& Falcke, 2015; Eriksson \& Mazerolle, 2015; Colossi \& Falcke, 2019; Marasca, Colossi, \& Falcke, 2013; Roberts, McLeughlin, Conron, \& Koenen, 2011; Timmons, Arbel, \& Margolin, 2017). Os dados evidenciam que vivenciar, na família de origem, o contexto de violência pode levar à tendência de repetição dos padrões nos relacionamentos adultos (Falcke, 2003; Mendlowicz \& Figueira, 2007).

Essa tendência possivelmente derive do fato de que pessoas com histórico de violência experienciada na família de origem estão mais predispostas a perceber a violência como um fenômeno esperado na relação conjugal, naturalizando-o (Temple, Shorey, Tortolero, Wolfe, \& Stuart, 2013). Nesse sentido, as experiências na família de origem parecem associar-se a ocorrência da violência conjugal, ainda que os estudos não especifiquem de forma detalhada qual tipo de violência pode ter maior poder explicativo e se existem outras variáveis que podem mediar essa relação, contribuindo para diferenciar os que repetem a violência daqueles que rompem o ciclo.

Considerando as experiências adversas na família de origem, diferentes dimensões foram foco de análise de estudos, especialmente dedicados a analisar como as experiências na família de origem impactam a saúde na vida adulta décadas mais tarde. Para melhor clarificar a conceituação e tipologia, foi construída uma tabela, classificando os conceitos em duas grandes categorias: abuso/negligência e disfuncionalidade familiar.

Os estudos, em geral, destacam os impactos negativos das experiências disfuncionais vivenciadas na família de origem para o desenvolvimento e os relacionamentos futuros. Todavia, ainda que o abuso/ negligência e a disfuncionalidade familiar se façam presentes nas experiências de família de origem, ao conhecer os aspectos transgeracionais é possível fazer escolhas mais conscientes do que se deseja. Nesse sentido, experiências passadas, ainda que, com frequência, repitam-se, também podem ser modificadas quando o sujeito reconhece suas conexões familiares (Colossi \& Falcke, 2019; Sant'Anna \& Penso, 2018).

A conjugalidade diz respeito a um processo interacional (Ozório, Féres-Carneiro, \& Magalhães, 2017), constituído a partir de dois passados que se unem, no qual muitas das condutas e atitudes dos cônjuges são motivadas a partir do processo de aprendizagem no ambiente familiar. Psicoterapeutas de casal descrevem que, muitas vezes, o novo casal apresenta dificuldade para se diferenciar de sua família de origem, principalmente no que diz respeito à criação de suas regras, seus valores, seus objetivos, ou seja, na criação de sua própria identidade (Schuster et al., 2009).

Um estudo de delineamento explicativo, com 2.500 universitários nos Estados Unidos, realizado por Gover, Kaukinen e Fox (2008) com objetivo de verificar a relação entre violência no namoro e família de origem, apontou que a exposição à violência durante a infância é um preditor para o envolvimento em relações violentas. Nesse sentido, pode-se mencionar que a relação que se estabelece entre as figuras maternas e paternas será determinante para a qualidade do vínculo conjugal (Schuster et al., 2009).

Spencer, Cafferky e Stith (2016) realizaram uma meta-análise, com 580 estudos, que indicavam 60 fatores de risco para ocorrência da violência conjugal, dividiram em quatro grupos $(1$ - dados demográficos, 2 - saúde mental e questões individuais, 3 - família de origem e 4 -fatores de risco no relacionamento) e compararam entre homens e mulheres. Como resultados, encontraram apenas três fatores de risco que diferenciaram homens e mulheres, sendo dois de família de origem (presenciar episódios de violência dos pais ou experimentar abuso físico na infância e padrões de comunicação) e um fator de saúde mental e questões individuais (abuso de álcool). Os autores recomendam que as pesquisas não devem focar no poder e controle do homem sobre a mulher, e sim nas estratégias de resolução de conflito do casal. Como limitação do estudo, os autores referiram não terem encontrado estudos suficientes para analisar outros fatores que podem impactar na ocorrência de violência, como ciúme, por exemplo.

O ciúme pode ser compreendido como uma ameaça relacionada ao medo (emoção) de perda (real ou imaginária) do parceiro, que é influenciada por sentimentos, como posse, amor e competitividade (Haack, 2017). Conforme a autora, ele está circunscrito a um determinado contexto cultural, social e histórico, ocorrendo em uma perspectiva individual e relacional, em níveis mais ou menos patológicos.

A literatura destaca a associação entre o ciúme e a ocorrência de episódios de violência na conjugalidade (Centeville \& Almeida, 2014; Lacerda \& Costa, 2014). O fenômeno é apontado como um dos principais 
Tabela 1.

Experiências de Familia de Origem Destrutivas

\begin{tabular}{|c|c|c|}
\hline Dimensão & Tipo & Conceito \\
\hline \multirow{5}{*}{ 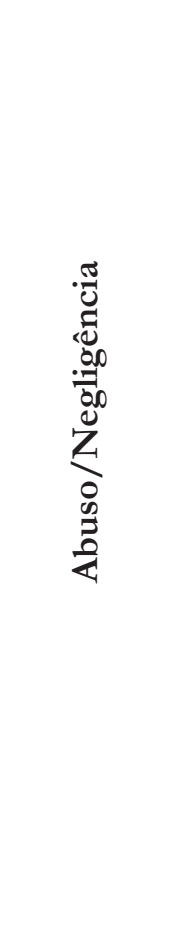 } & $\begin{array}{l}\text { Abuso físico (Carvalho \& Gomide, 2005; } \\
\text { Cecconello, De Antoni, \& Koller, 2003). }\end{array}$ & $\begin{array}{l}\text { Uso de força física de maneira intencional, com } \\
\text { objetivo de ferir, lesar ou destruir a criança ou } \\
\text { adolescente (Ministério da Saúde, 2001, 2002). }\end{array}$ \\
\hline & $\begin{array}{l}\text { Abuso emocional ou psicológico (Díaz Lemus, } \\
\text { Fornaris Díaz, \& Segredo Pérez, 2014; Russo, } \\
\text { Dantas, Nogueira, \& Trindade, 2014) }\end{array}$ & $\begin{array}{l}\text { Toda forma de rejeição, depreciação, } \\
\text { discriminação, desrespeito, cobranças exageradas, } \\
\text { punições humilhantes e utilização da criança } \\
\text { ou adolescente para atender as necessidades } \\
\text { psíquicas do adulto (Ministério da Saúde, 2001, } \\
\text { 2002). }\end{array}$ \\
\hline & $\begin{array}{l}\text { Abuso sexual (Deslandes, Mendes, \& Luz, 2014; } \\
\text { Risman, Figueira, Vieira, \& Azevedo, 2014). }\end{array}$ & $\begin{array}{l}\text { Ato ou jogo sexual, que tem intenção de } \\
\text { estimular sexualmente a criança ou adolescente, } \\
\text { utilizando-os para obter satisfação sexual } \\
\text { (Ministério da Saúde, 2001, 2002). }\end{array}$ \\
\hline & Negligência física (Russo et al., 2014). & $\begin{array}{l}\text { Omissão, deixando de prover necessidades } \\
\text { básicas para o desenvolvimento físico, como falta } \\
\text { de cuidados básicos, privação de não provimento } \\
\text { de estímulos e condições para a frequência à } \\
\text { escola (Ministério da Saúde, 2001,2002). }\end{array}$ \\
\hline & Negligência emocional (Russo et al., 2014). & $\begin{array}{l}\text { Falta de atenção, carinho, afeto e suporte } \\
\text { emocional (Ministério da Saúde, 2001, 2002). }\end{array}$ \\
\hline \multirow{3}{*}{ 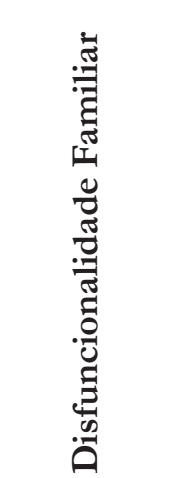 } & $\begin{array}{l}\text { Abuso de substâncias pelos cuidadores (Seleghim } \\
\text { \& Oliveira, 2013). }\end{array}$ & $\begin{array}{l}\text { Existência, no grupo familiar, de um abusador de } \\
\text { álcool ou drogas (Melchert, 1998). }\end{array}$ \\
\hline & $\begin{array}{l}\text { Ajustamento psicológico parental (Arcos et al., } \\
\text { 2013). }\end{array}$ & $\begin{array}{l}\text { Saúde mental dos pais, avaliando, por exemplo, } \\
\text { se existe alguém na família que sofra de } \\
\text { depressão crónica, doença mental ou tenha } \\
\text { comportamentos suicidas (Melchert, 1998). }\end{array}$ \\
\hline & $\begin{array}{l}\text { Aliança parental e violência interparental } \\
\text { (Benetti, 2006; Finger, Hans, Bernstein, \& Cox, } \\
\text { 2009). }\end{array}$ & $\begin{array}{l}\text { Qualidade do relacionamento conjugal dos } \\
\text { cuidadores e exposição dos filhos ao conflito } \\
\text { conjugal (Benetti, 2006). }\end{array}$ \\
\hline
\end{tabular}

desencadeadores de violência conjugal (Centeville \& Almeida, 2014; Cecchetto, Moreira Oliveira, Njaine, \& Minayo, 2016; Cortez, Cruz, \& Souza, 2014; Fonseca, Ribeiro, \& Leal, 2012; Machado et al., 2014) e também como um dos culpados pela banalização da violência (Amendola, 2005), uma vez que os agressores justificam que sentem ciúme e agridem porque amam muito o parceiro. Nesse sentido, é possível pensar que o ciúme poderia ser um fator mediador entre as experiências na família de origem e a ocorrência da violência conjugal, inclusive diferenciando aqueles que vivenciaram violência na família de origem e cometem em seus relacionamentos atuais daqueles que também a vivenciaram, mas não cometem.

Um estudo com o objetivo de conhecer as características sociodemográficas e de saúde das mulheres vítimas de violência que estiveram sob a tutela protetora do Estado do Ceará, com uma amostra composta de 197 prontuários de mulheres atendidas entre 2001 e 2012, indicou que o principal fator desencadeante das agressões foi o ciúme (Ferreira, Vasconcelos, Moreira-Filho, \& Macena, 2016). Além disso, o ciúme, juntamente com a vingança, é comumente associado aos motivos que levam à ocorrência de crimes passionais (Rufatto, 2016). 
Compreender de que forma as experiências familiares e o ciúme podem produzir efeitos na ocorrência de violência conjugal pode contribuir para o planejamento de futuras intervenções que auxiliem os casais na vivência de uma conjugalidade mais funcional. Nesse sentido, a partir da abordagem sistêmica, o objetivo desta pesquisa foi testar um modelo explicativo do ciúme como mediador entre as experiências na família de origem e a violência física conjugal.

\section{Método}

Trata-se de uma pesquisa de caráter quantitativo, transversal, que se caracteriza pelo delineamento explicativo.

\section{Participantes}

Participaram deste estudo 600 pessoas, 384 mulheres e 216 homens heterossexuais, com idade entre 18 e $65 \operatorname{anos}(M=31,69 ; D P=9,74)$. Residentes em 15 estados brasileiros e no Distrito Federal (Bahia, Ceara, Goiás, Mato Grosso do Sul, Minas Gerais, Paraíba, Paraná, Pernambuco, Piauí, Rio de Janeiro, Rio Grande do Sul, Rio Grande do Norte, Rondônia, São Paulo, Santa Catarina e Distrito Federal), o tempo de relacionamento variou entre 1 e 40 anos $(M=8,60 ; D P=$
8,30). Na Tabela 2, encontram-se características sociodemográficas da amostra.

\section{Instrumentos}

Questionário de dados sociodemográficos. Composto de 13 itens, teve como objetivo mapear algumas características dos participantes, como cidade, estado, situação amorosa, tempo de relacionamento, filhos, entre outros.

Subescalas do FBQ - Family Background Questionnaire (Melchert, 1998; Melchert \& Sayer, 1998). Esse questionário que tem por objetivo examinar o funcionamento da família de origem a partir da percepção dos sujeitos sobre suas experiências. É composto por 179 itens a serem respondidos em escala Likert de cinco pontos. Neste estudo, foram utilizados 62 itens correspondentes às subescalas de negligência física, abuso físico paterno e materno, abuso sexual, abuso de substâncias paterno e materno, ajustamento psicológico paterno e materno e aliança parental. As pontuações são cotadas de maneira que as mais elevadas indicam um melhor nível de funcionamento familiar.

Considerando as subescalas, negligência física consiste na falta de cuidados físicos, como de alimentação, vestuário, condições de higiene e outros. Abuso físico paterno e materno averigua um continum disfuncional que inclui violência física e abuso. Abuso sexual

Tabela 2.

Frequências e Percentuais das Características Sociodemográficas da Amostra $(n=600)$

\begin{tabular}{|c|c|c|c|}
\hline \multicolumn{2}{|c|}{ Características } & $\mathrm{N}$ & $\%$ \\
\hline \multirow{4}{*}{ Situação conjugal } & Namorando & 215 & 35,8 \\
\hline & Morando juntos/União & 179 & 29,8 \\
\hline & estável & 206 & 34,3 \\
\hline & Casados oficialmente & & \\
\hline \multirow[b]{2}{*}{ Filhos } & Sim & 184 & 30,8 \\
\hline & Não & 414 & 69,2 \\
\hline \multirow{5}{*}{ Escolaridade } & Sem instrução & 1 & 2 \\
\hline & Fundamental & 12 & 2 \\
\hline & Médio & 219 & 37 \\
\hline & Superior & 202 & 34,1 \\
\hline & Pós graduação & 158 & 26,7 \\
\hline \multirow{2}{*}{$\begin{array}{l}\text { Exerce atividade } \\
\text { remunerada }\end{array}$} & Sim & 482 & 80,9 \\
\hline & Não & 114 & 19,1 \\
\hline
\end{tabular}


avalia a vivência de atos sexuais em que não existe contato físico (como voyeurismo, exibicionismo) até sexo com ou sem penetração, cometidos por pai, mãe, parentes ou pessoas próximas e desconhecidos. Abuso de substâncias paterno e materno avalia o uso de substâncias químicas (álcool ou drogas) por parte dos pais e o grau de abuso. O ajustamento psicológico refere-se à saúde mental dos pais, especificamente perturbações de humor ou consumo de substâncias. Por fim, aliança parental é o grau de acordo entre o pai e a mãe em relação as regras ou instruções aos filhos e a avaliação do relacionamento conjugal dos pais.

A escala foi traduzida para o português, tendo obtido bons índices de confiabilidade de 0,99 para a escala total e entre 0,40 e 0,95 para as subescalas (Falcke, 2003). Neste estudo, obteve-se os seguintes alfas: 0,73 negligência física; 0,84 abuso físico paterno e 0,79 materno, 0,31 abuso sexual; 0,89 abuso de substâncias paterno e 0,82 materno; 0,77 ajustamento psicológico paterno e 0,76 materno; 0,83 aliança parental e 0,90 escala total.

Subescalas da CTS 2 - Revised Conflict Tactics Scale (Straus, Hamby, Buncy-McCoy, \& Sugarman, 1996). Criada para verificar violência entre pessoas que tenham uma relação de namoro, casamento ou afins. Contém 78 itens que compõem cinco dimensões: violência física, agressão psicológica, coerção sexual, lesão corporal e negociação. Para fins deste estudo, foram utilizadas apenas as subescalas de violência fisica e lesão corporal cometidas pelo sujeito. A violência física é definida por Strauss (2008) como agressões menores (empurrar, agarrar, dar tapa, jogar objeto, torcer o braço e puxar o cabelo) e graves (dar soco, bater, chutar, jogar contra a parede, queimar ou escaldar, usar uma faca ou arma de fogo). Lesão corporal menor corresponde a causar torção, contusão, mancha roxa ou dores no corpo que duraram até o dia seguinte em função de uma briga, enquanto que a lesão corporal grave diz respeito a levar o(a) parceiro(a) a ter a necessidade de busca por auxílio médico após uma briga ou ocorrência de desmaio e quebra de algum osso. É apresentada em um modelo de checklist, conforme a frequência das situações apresentadas. Foi utilizada a versão brasileira adaptada e traduzida de Moraes, Hasselman e Reichenheim (2002). Neste estudo, obteve-se os seguintes alfas: 0,68 violência fisica grave; 0,76 violência fisica menor; 0,54 lesão corporal grave; 0,75 lesão corporal menor.

ICR - Inventário de Ciúme Romântico (Bueno et al., 2012). Tem por objetivo descrever a ocorrência de reações de ciúme do participante com relação aos comportamentos do cônjuge. Ele é composto de 41 itens, respondidos em uma escala do tipo Likert de cinco pontos. É composto por seis fatores: o F1 (não contato com o parceiro) diz respeito às crenças ou aos pensamentos de infidelidade quando não consegue contato com o parceiro ou ante a perda de controle sobre sua localização. O F2 (contato parceiro-rival) se refere às reações emocionais típicas do ciúme com relação à percepção do parceiro em um relacionamento com um possível rival. Reações agressivas direcionadas ao parceiro compõe o F3 (agressão ao parceiro) e relacionadas ao rival compõe o F4 (agressão ao rival). O fator 5 (autoestima) descreve reações no qual o indivíduo se atribui à culpa pela possibilidade de infidelidade do parceiro. Por fim, o fator 6 (investigação) se refere a comportamentos de investigação relacionadas à fidelidade do parceiro. Neste estudo, obteve-se os seguintes alfas: 0,92 F1; 0,87 F2; 0,87 F3; 0,70 F4; 0,66 F5; 0,65 F6 e 0,92 na escala total.

\section{Procedimentos}

O estudo foi realizado por meio de um formulário eletrônico, que foi disponibilizado aos participantes por meio de um endereço eletrônico. O processo de amostragem ocorreu de forma intencional, por meio do envio de convites por meio de redes sociais. Também foi considerado o critério de amostragem por bola de neve, uma vez que foi solicitado que os participantes encaminhassem o convite para os seus conhecidos por meio de e-mail ou mensagens. Na primeira tela o participante teve acesso ao Termo de Consentimento Livre e Esclarecido (TCLE), que exigiu uma resposta obrigatória.

A presente pesquisa seguiu as orientações das Resoluções 466/2012 do Conselho Nacional de Saúde (2012), sobre pesquisas com seres humanos. Esta pesquisa faz parte de um projeto maior intitulado "Ciúme, infidelidade e violência conjugal", financiado pelo Conselho Nacional de Desenvolvimento Científico e Tecnológico (CNPq), que foi aprovado pelo Comitê de Ética da Universidade do Vale do Rio dos Sinos, sob o protocolo 13/182. Os dados referentes à identificação de todos os participantes da pesquisa, como endereço de IP e e-mail (para aqueles que solicitarem a devolução da pesquisa) foram mantidos em sigilo e em nenhum momento serão divulgados.

\section{Análise dos Dados}

Os dados foram analisados inicialmente no programa SPSS 22.0 (Statistical Package for Social Science), 
primeiramente por meio de estatísticas descritivas para calcular as médias, o desvio padrão e análises de correlações. Após isso, foi utilizado o programa AMOS 20.0, no qual, a partir das análises obtidas, foram realizadas análises fatoriais confirmatórias e a Modelagem de Equação Estrutural (MEE). O modelo proposto foi composto por três variáveis latentes: experiências na família de origem (variável independente e exógena), ciúme (variável dependente, mediadora, endógena e exógena) e violência conjugal (variável dependente, endógena). O modelo utilizado é recursivo (Hair, Black, Babin, Anderson, \& Tatham, 2009) que têm como peculiaridade a ausência de covariância entre distúrbios de variáveis endógenas e a unidirecionalidade das relações de determinação entre as variáveis. $\mathrm{O}$ método de estimação utilizado foi o de Máxima Verossimilhança (Maximum Likelihood - MLE) que estima os parâmetros que maximizam a verossimilhança ao observar a matriz de covariância amostral (Marôco, 2010). A análise de adequação do modelo de equações estruturais usou as seguintes medidas de ajuste (Hair et al., 2009; Marôco, 2010): Qui-Quadrado $\left(\chi^{2}\right)$, significância estatística de $\chi^{2}(p)$, raiz do erro quadrático médio de aproximação (RMSEA), índice de Tucker-Lewis (TLI), índice de ajuste ponderado (NFI), índice de ajuste comparativo (CFI) e Qui-Quadrado ponderado $\left(\chi^{2} / d f\right)$.

\section{Resultados}

Com relação à família de origem, a maioria dos participantes conviveu com os pais durante toda a infância $(91,2 \%)$ e estes permaneceram casados e/ou mantiveram-se juntos até algum deles falecer (72,9\%). Metade dos participantes não recorda de situações de ciúme entre os pais (50\%); $12 \%$ referem que o pai era ciumento com a mãe; $21 \%$ descrevem que a mãe era ciumenta com o pai e $17 \%$ recordam que ambos eram ciumentos.

Ainda com relação ao ciúme, $50,5 \%$ se consideram um pouco ciumentos, $13,3 \%$ se consideram muito ciumentos e 36,2\% afirmam não sentirem ciúme. Questionados sobre a frequência que sentem ciúme, $3,7 \%$ respondeu que sempre; $19,7 \%$ frequentemente; $71,5 \%$ raramente e 5,2\% que nunca sentem. Quando questionados se o ciúme ocasionava prejuízos ao relacionamento, 39,8\% responderam que sim.

Ainda sobre o relacionamento conjugal, observou-se, com relação à ocorrência da violência os seguintes índices: $7,3 \%$ violência física grave; $28,3 \%$ violência física menor; $2,5 \%$ lesão corporal grave e 7,8\% lesão corporal menor. As correlações entre todas as variáveis alternam entre moderadas a fracas e podem ser observadas na Tabela 3 .

Considerando as correlações entre ciúme, violência e família de origem, conforme se observa na Tabela 3, optou-se em não inserir no modelo as variáveis que não se correlacionaram com a maioria (quatro fatores ou mais) da escala de ciúme. Ainda que a variável ajustamento psicológico paterno tenha entrado no critério de associação ao ciúme, não se correlacionou com a violência física grave $(r=-0,022 ; p=0,596)$, lesão corporal grave $(r=0,021 ; p=0,608)$ e menor $(r=0,076$; $p=$ $0,065)$, portanto não foi utilizada. Dessa forma, apenas foram utilizadas as variáveis abuso sexual, negligência física e aliança parental no que se refere às experiências na família de origem.

O modelo estrutural proposto baseou-se na hipótese de que o ciúme media as experiências na família de origem e a ocorrência da violência conjugal. Os resultados de ajuste do modelo são mostrados na Tabela 4.

O Modelo 1 mostrou resultados inadequados para um modelo de equação estrutural, porque apresentou valores insuficientes de TLI e NFI e resíduos altos. No Modelo 2, foi ajustada a covariância entre violência física grave e a violência física menor. Com isso, o modelo apresentou resultados satisfatórios. $\mathrm{O}$ ajuste final pode ser observado na Figura 1.

Ao examinar os coeficientes padronizados de regressão do modelo, identifica-se que a magnitude da predição das experiências de família de origem para o ciúme é fraca negativa $(\beta=-0,25 ; p<0,001)$, que, por sua vez, impacta de maneira positiva e fraca na violência conjugal $(\beta=0,47 ; p<0,001)$. O caminho direto entre as experiências na família de origem e a violência conjugal se manteve com magnitude negativa e fraca $(\beta=$ $-0,25 ; p<0,001)$.

A mediação facilita a relação entre dois construtos envolvidos e exige correlação significativa entre eles individualmente. Para verificar a extensão do efeito mediador do modelo de acordo com Hair, Black, Babin, Anderson e Tatham (2009), algumas análises devem ser realizadas. O primeiro passo é verificar individualmente se o tamanho de efeito direto entre os construtos latentes foi significativo. Assim identificou-se o impacto entre: experiências na família de origem e ciúme $(\beta=-0,25 ; p$ $<0,001)$; ciúme e violência $(\beta=0,46 ; p<0,001)$ e experiências na família de origem e violência $(\beta=-0,24 ; p$ $<0,001)$. Posteriormente, analisou-se o relacionamento das variáveis no modelo. A relação entre experiências 
Tabela 3.

Correlações entre Ciúme, Violência e Experiências na Família de Origem

\begin{tabular}{|c|c|c|c|c|c|c|c|}
\hline 节 & & $\begin{array}{c}\text { Não contato } \\
\text { com o } \\
\text { parceiro }(\mathrm{F} 1)\end{array}$ & $\begin{array}{c}\text { Contato } \\
\text { parceiro-rival } \\
\text { (F2) }\end{array}$ & $\begin{array}{l}\text { Agressão ao } \\
\text { parceiro (F3) }\end{array}$ & $\begin{array}{l}\text { Agressão ao } \\
\text { rival (F4) }\end{array}$ & $\begin{array}{l}\text { Autoestima } \\
\text { (F5) }\end{array}$ & $\begin{array}{c}\text { Investigação } \\
\text { (F6) }\end{array}$ \\
\hline \multirow{4}{*}{ 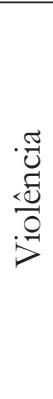 } & $\begin{array}{l}\text { Violência Física } \\
\text { Grave }\end{array}$ &, $261^{* *}$ &, $166^{* *}$ &, $357^{* *}$ & $207^{* *}$ & ,103* &, $341^{* *}$ \\
\hline & $\begin{array}{l}\text { Violência Física } \\
\text { Menor }\end{array}$ &, $375^{* *}$ &, $302^{* *}$ &, $493^{* *}$ &, $355^{* *}$ &, $152^{* *}$ &, $437^{* *}$ \\
\hline & $\begin{array}{l}\text { Lesão Corporal } \\
\text { Grave }\end{array}$ &, $115^{* *}$ &, 075 &, $156^{* *}$ &, $100^{*}$ & ,027 &, $187^{* *}$ \\
\hline & $\begin{array}{l}\text { Lesão Corporal } \\
\text { Menor }\end{array}$ &, $215^{* *}$ &, $147^{* *}$ &, $316^{* *}$ &, $155^{* *}$ & ,070 &, $257^{* *}$ \\
\hline \multirow{9}{*}{ 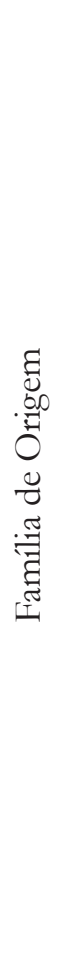 } & $\begin{array}{l}\text { Abuso físico } \\
\text { paterno }\end{array}$ & ,006 &,- 027 &,- 038 &,- 050 &,- 067 &,- 045 \\
\hline & $\begin{array}{l}\text { Abuso físico } \\
\text { materno }\end{array}$ &,- 031 & ,001 &,$- 080^{*}$ &,- 041 &,- 073 &,$- 114^{* *}$ \\
\hline & Abuso sexual &, $110^{* *}$ & ,024 &, $088^{*}$ & ,071 &, $106^{* *}$ &, $135^{* *}$ \\
\hline & $\begin{array}{l}\text { Negligência } \\
\text { física }\end{array}$ &,$- 092^{*}$ &,- 021 &,$- 134^{* *}$ &,$- 150^{* *}$ &,$- 137^{* *}$ &,$- 130^{* *}$ \\
\hline & $\begin{array}{l}\text { Abuso de } \\
\text { substancias } \\
\text { paterno }\end{array}$ &,- 040 &,- 009 &,- 020 &,- 016 & ,003 &,- 057 \\
\hline & $\begin{array}{l}\text { Abuso de } \\
\text { substancias } \\
\text { materno }\end{array}$ & ,025 & ,051 & ,034 & ,002 & ,015 & ,013 \\
\hline & $\begin{array}{l}\text { Ajustamento } \\
\text { psicológico } \\
\text { paterno }\end{array}$ &,$- 102^{*}$ &,- 075 &,$- 156^{* *}$ &,$- 127^{* *}$ &,- 073 &,$- 131^{* *}$ \\
\hline & $\begin{array}{l}\text { Ajustamento } \\
\text { psicológico } \\
\text { materno }\end{array}$ &,$- 094^{*}$ &,- 052 &,$- 121^{* *}$ &,- 080 &,- 069 &,$- 154^{* *}$ \\
\hline & Aliança parental &,$- 164^{* *}$ &,$- 103^{*}$ &,$- 197^{* *}$ &,$- 158^{* *}$ &,$- 133^{* *}$ &,$- 164^{* *}$ \\
\hline
\end{tabular}

**. A correlação é significativa no nível 0,01 (2 extremidades).

*. A correlação é significativa no nível 0,05 (2 extremidades).

na família de origem e violência reduziu o tamanho de efeito quando a variável latente de ciúme foi incluída no modelo. Esse dado indica mediação parcial do ciúme no modelo, pois a relação direta se manteve significativa, entretanto foi reduzida $(\beta=-0,36 ; p<0,001$ para $\beta=$ $-0,25 ; p<0,001)$ (Hair et al., 2009).

O modelo teórico inicial supôs que o ciúme mediaria as relações entre as experiências na família de origem e a ocorrência da violência conjugal. A hipótese foi confirmada, indicando a existência de caminhos diretos e indiretos entre os construtos, o que caracteriza a existência de mediação parcial entre as experiências na família de origem e a ocorrência da violência conjugal.

\section{Discussão}

Os dados descritivos da amostra chamam a atenção especialmente quando os participantes responderam 
Tabela 4.

Teste de Ajuste do Modelo

\begin{tabular}{llllllll}
\hline & $\chi^{2}$ & $p$ & $\chi^{2} / d f$ & RMSEA & CFI & TLI & NFI \\
\hline Modelo 1 & 357,959 &, 000 & 5,77 &, 089 &, 908 &, 884 &, 891 \\
Modelo 2 & $\mathbf{1 9 2 , 0 3 6}$ & $\mathbf{, 0 0 0}$ & $\mathbf{3 , 1 4}$ & $\mathbf{, 0 6 0}$ & $\mathbf{, 9 5 9}$ & $\mathbf{, 9 4 8}$ & $\mathbf{9 4 2}$ \\
\hline
\end{tabular}

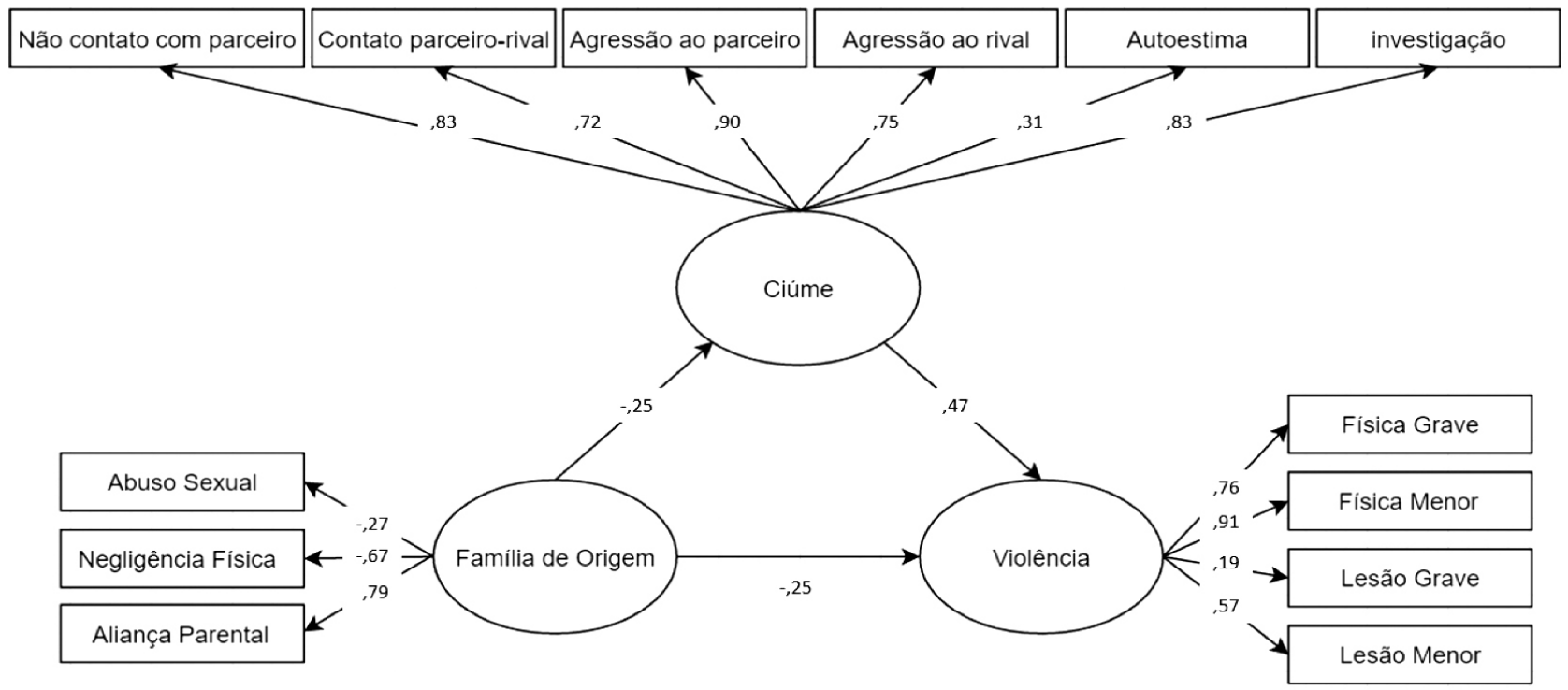

Figura 1. Modelo final de violência.

diretamente sobre o quanto se consideravam ciumentos. Inicialmente, $36,2 \%$ da amostra declarou não sentir ciúmes, mas, ao mesmo tempo, quando questionados sobre a frequência com que sentiam ciúme, apenas $5,2 \%$ da amostra declarou nunca sentir. Uma hipótese para esse achado é o fato do conceito de ciúme ser muito abrangente e depender das crenças de cada sujeito (Carvalho \& Ambiel, 2016). Além disso, como temática frequente no cotidiano popular, assim como em músicas, poesias e na literatura brasileira, o ciúme é descrito como prova de amor e de cuidado, sendo interpretado social e/ou culturalmente como um sentimento de zelo (Costa et al., 2014). Nesse sentido, pode ser um fenômeno naturalizado nas relações íntimas, mascarando, por vezes, a gravidade de situações levam a ocorrência da violência conjugal.

Outro fator que merece destaque é o fato de que metade da amostra identificou ciúme no relacionamento dos pais, o que pode sugerir o impacto da transmissão transgeracional. Corroborando essa hipótese, Turatti e Lucas (2016) encontraram relação entre os comportamentos de ciúme dos pais influenciando na relação amorosa dos filhos.

Com relação à agressão física grave e menor, os índices obtidos nesta pesquisa foram maiores em comparação com os estudos de Bolze, Crepaldi, Schmidt e Vieira (2013) e Madalena, Falcke e Carvalho (2015) e menores em comparação com a ocorrência de lesão corporal maior e menor. Entretanto, cabe salientar que, embora existam diferenças entre estudos na porcentagem da ocorrência da violência, medida pelo mesmo instrumento de pesquisa, ela é pequena, podendo ser atribuída a diferenças amostrais. Em todos os casos, esses índices de violência conjugal merecem atenção porque se configuram como um problema de grande magnitude, que afeta não somente a díade, mas todos os membros da família (Vian, Mosmann, \& Falcke, 2019).

Muitas associações foram encontradas entre as experiências na família de origem, o ciúme e a violência conjugal. Considerando as experiências na família de origem, as variáveis que compuseram o construto foram aliança parental, negligência física dos pais com 
relação aos cuidados dos filhos e abuso sexual. Pode-se pensar, considerando os resultados, na tendência à repetição dos padrões familiares, uma vez que a disfuncionalidade no relacionamento conjugal dos pais e frente aos cuidados dos filhos pode gerar inseguranças, ocorrendo a repetição de falta de cuidados no relacionamento íntimo. Esses comportamentos podem dar margem para ocorrência de comportamentos ciumentos, uma vez que tendem a gerar insegurança frente a necessidades não atendidas. Vivenciar experiências traumáticas na infância pode gerar dificuldades na idade adulta, como, por exemplo, no estabelecimento e a manutenção de relações saudáveis (Krindges, Macedo, \& Habigzang, 2016; Staples, Rellini, \& Roberts, 2012; Turchik, 2012). Os resultados corroboram os achados de Colossi, Marasca e Falcke (2015), quando descrevem que pais que apresentam características mais disfuncionais em seus relacionamentos íntimos, possuem mais chances de terem filhos que utilizem modelos de relacionamentos baseados em diferentes expressões de violência. E ainda, pais que apresentam maior ajustamento conjugal, coesão e flexibilidade proporcionam aos filhos configurações mais adaptativas e funcionais na transgeracionalidade.

A literatura aponta que as sequelas produzidas devido a problemas na infância podem causar danos ao desenvolvimento físico, emocional e social, que podem durar anos, gerando efeitos devastadores (Egry, Apostólico, Albuquerque, Gessner, \& Fonseca, 2015). Legitimando, Gomes (2015) descreve que a repetição de experiências disfuncionais agrava suas consequências ao longo do tempo, necessitando a identificação precoce de seus sinais e uma rede de apoio social de proteção à criança e à família.

No mesmo sentido, o ciúme pode gerar sérias consequências no estabelecimento e manutenção dos relacionamentos. A literatura indica que pessoas ciumentas são mais inseguras e possuem como característica problemas relacionados à autoestima, o que pode fazer com que a pessoa que sente ciúme se anule no relacionamento, na tentativa de agradar o parceiro. Dessa forma, o ciúme tem levado ao fracasso inúmeros relacionamentos, que muitas vezes acabam em brigas e discussões (Turatti \& Lucas, 2016), expressando-se inclusive em violência física e lesão corporal.

Sendo assim, os achados desta pesquisa confirmam relações previamente descritas na literatura de que as experiências familiares impactam a ocorrência da violência conjugal (Marasca, Colossi, \& Falcke, 2013; Roberts et al., 2011), possivelmente causando riscos à qualidade de vida dos envolvidos (Barreto et al., 2016). Por meio da confirmação da mediação parcial do ciúme na ocorrência de violência física nessas relações, os resultados corroboram os achados de estudos prévios de que experiências na família de origem e ciúme encontram-se entre os principais desencadeadores de violência conjugal (Cecchetto et al., 2016; Centeville \& Almeida, 2014; Cortez, Cruz, \& Souza, 2014; Fonseca, Ribeiro, \& Leal, 2012; Machado et al., 2014).

O modelo explicativo testado do ciúme como mediador entre as experiências na família e origem e a ocorrência da violência física conjugal foi satisfatório, desse modo, o artigo contribui para um maior reconhecimento dos aspectos intrínsecos ao fenômeno da violência conjugal e seus fatores de risco. Indica que, ainda que a pessoa tenha vivenciado experiências precoces de violência na família de origem e de ciúmes no relacionamento atual, pode aumentar ou diminuir as chances de repetição dos padrões de violência nestes. Nesse sentido, esses dados servem de auxílio para profissionais, médicos, enfermeiros, assistentes sociais e psicólogos como alerta de como o ciúme pode contribuir para o desencadeamento de agressões que geram graves consequências as vítimas, demandando atenção às demonstrações inicias de ciúme.

É de extrema importância que não se olhe para o fenômeno de maneira isolada, apenas para questões aparentes como as marcas que uma possível agressão física ou lesão corporal podem deixar. É essencial que seja dada visibilidade, nas situações de violência conjugal, a fenômenos que podem estar ocultos, como as experiências na família de origem e o ciúme, que muitas vezes se mantêm velados. Além disso, cuidado também deve ser tomado com a concepção de que o ciúme pode ser demonstração de carinho ou afeto, o que pode dificultar o reconhecimento precoce de agravos da violência, naturalizando o fenômeno.

Torna-se fundamental, acima de tudo, um maior investimento na prevenção desses fenômenos, identificando possíveis episódios nas crianças enquanto vivenciam suas experiências na família de origem e, assim, quem sabe, evitando que estas se tornem pessoas inseguras e ciumentas, prevenindo possível repetição de violência na idade adulta e contribuindo assim para um desenvolvimento mais saudável. Trabalhar os processos de separação-individuação em relação à família de origem (Bowen, 1991) pode ser aspecto chave para que a pessoa reconheça suas vivências passadas e seus sentimentos atuais motivados por elas, como ciúme, por exemplo, conseguindo diferenciar-se e agir como 
um indivíduo mais autônomo para fazer suas próprias escolhas, e não seguir repetindo os padrões familiares aprendidos.

Esta pesquisa possui como limitação características da amostra, como a maioria dos sujeitos sem filhos, namorando ou com alta escolaridade e seu caráter transversal, o que pode prejudicar aspectos subjetivos desses casos, mas lança subsídios para identificar de antemão possíveis situações que possam levar a ocorrência da violência conjugal. Sugere-se que outras pesquisas sejam realizadas abarcando traços de personalidade, novas configurações conjugais e com a díade, com o objetivo de melhor compreensão das nuances existentes na conjugalidade e suas complexidades.

\section{Referências}

Amendola, G. (2005). Assassinos sem a menor importância: A banalização da violência no Brasil. São Paulo: Mostarda editora.

Arcos, E., Muñoz, L. A., Sanchez, X., Vollrath, A., Gazmuri, P., \& Baeza, M. (2013). Efetividade do sistema de proteção integral da infância em mães e crianças vulneráveis. Revista Latino-Americana de Enfermagem, 21(5), 1071-1079.

Barreto, M. D. S., Teston, E. F., Latorre, M. D. R. D. D., Mathias, T. A. D. F., \& Marcon, S. S. (2016). Mortalidade por acidentes de trânsito e homicídios em Curitiba, Paraná, 1996-2011. Epidemiologia e Serviços de Saúde, 25(1), 95-104. doi: 10.5123/ s1679-49742016000100010

Benetti, S. P. C. (2006). Conflito conjugal: Impacto no desenvolvimento psicológico da criança e do adolescente. Psicologia: Reflexão e Crítica, 19(2), 261-268. doi: 10.1590/s0102-79722006000200012

Bolze, S. D. A., Crepaldi, M. A., Schmidt, B., \& Vieira, M. L.. (2013). Marital Relationship and Tactics of Conflict Resolution between Couples. Actualidades en psicología, 27(114), 71-85. doi: 10.15517/ ap.v27i114.4828

Bowen, M. (1991). De la familia al individuo: La diferenciación del sí mismo en el sistema familiar. Buenos Aires: Paidós.

Brasil. Ministério da Saúde. Secretaria de Políticas de Saúde. (2001). Violência intrafamiliar: Orientações para a prática em serviço. Brasília: Ministério da Saúde, 2001. (Caderno de Atenção Básica, 8).
Brasil. Ministério da Saúde. Secretaria de Assistência à Saúde. (2002). Notificação de maus-tratos contra crianças $e$ adolescentes: Um passo a mais na cidadania em saúde. 2. ed. Brasília: Ministério da Saúde.

Bueno, J. M. H, Carvalho, L. F., Moreira, A. J. F., Capelo, A. M. A. B, Fernandes, A. M. C., Vasconcelos, M. F. G., ... et al., (2012). Adaptação do Inventário de Ciúme Romântico (ICR) para a população Portuguesa. Psico-USF, 17(3), 397-406. doi: 10.1590/ s1413-82712012000300006

Carvalho, L. F., \& Ambiel, R. A. M.. (2016). Diferenças entre os sexos no ciúme romântico: Um estudo brasileiro. Avances en Psicología Latinoamericana, 34(1), 143-155. doi: 10.12804/apl34.1.2016.10

Carvalho, M. C. N. D., \& Gomide, P. I. C. (2005). Práticas educativas parentais em famílias de adolescentes em conflito com a lei. Estudos de psicoogia, 22(3), 263-275. doi: 10.1590/s0103-166×2005000300005

Cecconello, A. M., De Antoni, C., \& Koller, S. H. (2003). Práticas educativas, estilos parentais e abuso físico no contexto familiar. Psicologia em estudo, 8, 45-54. doi: 10.1590/s1413-73722003000300007

Cecchetto, F., Moreira Oliveira, Q. B., Njaine, K., \& Minayo, M. C. S. (2016). Violências percebidas por homens adolescentes na interação afetivo-sexual em dez cidades brasileiras. Interface-Comunicação, Saúde, Educação, 20(59).doi: 10.1590/1807-57622015.0082

Centeville, V., \& de Almeida, T. (2014). Ciúme romântico e a sua relação com a violência. Psicologia Revista, 16(1/2), 73-91. Recuperado de https:// revistas.pucsp.br/index.php/psicorevista/article/ view $/ 18058$

Colossi, P. M., Marasca, A. R., \& Falcke, D. (2015). De geração em geração: A violência conjugal e as experiências na família de origem. Psico, 46(4), 493-502. Recuperado de http://revistaseletronicas.pucrs.br/ojs/index.php/revistapsico/article/ view/20979/14101

Colossi, P. M., Razera, J., Haack, K.R., \& Falcke, D. (2015). Violência conjugal: Prevalência e fatores associados. Contextos Clinicos, 8(1), 55-66. doi: doi. org/10.4013/ctc.2015.81.06

Conselho Nacional de Saúde (2012). Resolução 466 de 12 de Dezembro. Resolução no 466/2012, de 12 de dezembro de 2012. Brasília, DF: Autor. 
Cortez, M. B., Cruz, G. V., \& Souza, L. (2014). Violência conjugal: Desafios e propostas para a aplicação da Lei Maria da Penha. Psico, 44(4), 499-507. Recuperado de http://revistaseletronicas.pucrs.br/ojs/index.php/revistapsico/article/ view/11542/10842

Costa, N., Almeida, C., Gomes, H., Lobato, J., Gondim, L., Silva, M., Pinheiro, R. S., Almeida, T., \& Lima, V. A.. (2014). O ciúme está relacionado ao amor? Contribuições de uma perspectiva analítico-comportamental. Perspectivas em análise do comportamento, 5(1), 40-48. doi: 10.18761/perspectivas.v5i1.127

Deslandes, S. F., Mendes, C. H. F., \& Luz, E. S. (2014). Análise de desempenho de sistema de indicadores para o enfrentamento da violência intrafamiliar e exploração sexual de crianças e adolescentes. Revista Ciência \& Saúde Coletiva, 19(3), 865-874. doi: 10.1590/1413-81232014193.06012013

Díaz Lemus, M., Fornaris Díaz, E., \& Segredo Pérez, A. M. (2014). Violencia intrafamiliar en estudiantes de primer año de la Escuela Emergente de Enfermería. Revista Cubana de Medicina General Integral, 30(1), 113-120. Recuperado de http:// scielo.sld.cu/scielo.php?script=sci_arttext\&pi$\mathrm{d}=$ S0864-21252014000100011

Egry, E. Y., Apostólico, M. R., Albuquerque, L. M., Gessner, R., \& da Fonseca, R. M. G. S. (2015). Compreendendo a negligência infantil na perspectiva de gênero: Estudo em um município brasileiro. Revista da Escola de Enfermagem da USP, 49(4), 556563. Recuperado de http://www.scielo.br/pdf/ reeusp/v49n4/pt_0080-6234-reeusp-49-04-0556. pdf

Eriksson, L., \& Mazerolle, P. (2015). A cycle of violence? Examining family-of-origin violence, attitudes, and intimate partner violence perpetration. Journal of interpersonal violence, 30(6), 945-964.

Falcke, D. (2003). Águas passadas não movem moinhos? As experiências na família de origem como preditoras da qualidade do relacionamento conjugal (Tese de doutoramento). Universidade Federal do Rio Grande do Sul, Porto Alegre.

Falcke, D., \& Colossi, P. M. (2019). Implications of experiences in the Family of origin and infidelity in violence loving relashionships. Trends in Psychology, 27(2), 339-355.
Ferreira, R. M., Vasconcelos, T. B. de, Moreira Filho, R. E., \& Macena, R. H. M. (2016). Características de saúde de mulheres em situação de violência doméstica abrigadas em uma unidade de proteção estadual. Ciência \&o Saúde Coletiva, 21(12), 39373946. doi: 10.1590/1413-812320152112.09092015

Finger, B., Hans, S. L., Bernstein, V. J., \& Cox, S. M. (2009). Parent relationship quality and infant-mother attachment. Attachment and buman development, 11(3), 285-306. doi: 10.1080/14616730902814960

Fonseca, D. H., Ribeiro, C. G., \& Leal, N. S. B. (2012). Violência doméstica contra a mulher: Realidades e representações sociais. Psicologia \& Sociedade, 24(2), 307-314. doi: 10.1590/s0102-71822012000200008

Gomes, C. (2015). Maus tratos: A negligência parental $e$ a proteção social dos menores (Trabalho de conclusão de curso). Universidade Fernando Pessoa. PORTO. Recuperado de https://bdigital.ufp.pt/ bitstream/10284/6630/1/DM_Ana\%20Gomes. pdf

Gover, A. R., Kaukinen, C., \& Fox, K. A. (2008). The relationship between violence in the family of origin and dating violence among college students. Journal of Interpersonal Violence, 23(12),1667-1693. doi: $10.1177 / 0886260508314330$

Haack, K. R. (2017). Experiências na família de origem, ciüme e violência conjugal (Tese de doutorado em Psicologia). Programa de Pós-graduação em Psicologia, Universidade do Vale do Rio dos Sinos (UNISINOS), São Leopoldo. 97p.

Hair, J. J. F., Black, W. C., Babin, B. J., Anderson, R. E., \& Tatham, R. L. (2009). Análise multivariada de dados (6a. ed.). Grupo A - Bookman. Recuperado de http://public.eblib.com/choice/publicfullrecord. aspx?p=3235527

Hirigoyen, M. F. (2006). A violência no casal: Da coação psicológica à agressão física. Rio de Janeiro: Bertrand Brasil.

Krindges, C. A., Macedo, D. M., \& Habigzang, L. F. (2016). Abuso sexual na infância e suas repercussões na satisfação sexual na idade adulta de mulheres vítimas. Contextos Clínicos, 9(1), 60-71. doi: 10.4013/ctc.2016.91.05

Lacerda, L., \& Costa, N. (2014). Relação entre comportamentos emocionais ciumentos e violência contra a mulher. Revista Brasileira de Terapia Comportamental 
e Cognitiva, 15(3), 21-36. doi: 10.31505/rbtcc. v15i3.628

Machado, J. C., Rodrigues, V. P., Vilela, A. B. A., Simões, A. V., Morais, R. L. G. L., \& Rocha, E. N. (2014). Violência intrafamiliar e as estratégias de atuação da equipe de Saúde da Família. Saúde e Sociedade, 23(3), 828-840. doi: 10.1590/s0104-12902014000300008

Madalena, M. B. D. A., Falcke, D., \& Carvalho, L. D. F. (2015). Violência conjugal e funcionamentos patológicos da personalidade. Arquivos Brasileiros de Psicologia, 67(2). Recuperado de http://pepsic. bvsalud.org/scielo.php?script=sci_arttext\&pi$\mathrm{d}=$ S1809-52672015000200010

Marasca, A. R., Colossi, P. M., \& Falcke, D. (2013). Violência conjugal e família de origem: Uma revisão sistemática da literatura de 2006 a 2011. Temas em Psicologia, 21(1), 221-243. doi: 10.9788/tp2013.1-16

Marôco, J. (2010). Análise de equações estruturais Fundamentos teóricos, software e aplicações. Report Number, Pêro Pinheiro.

Melchert, T. P. (1998). Testing the validity of an instrument for assessing family of origin history. Journal of Clinical Psychology, 54, 863-875. doi: 10.1002/(sici)1097-4679(199811)54:7<863::aidjclp1>3.0.co;2-g

Melchert, T. P., \& Sayer, T. V. (1998). The development of an instrument for measuring memories of family of origin characteristics. Educational and Psychological Measurement, 58, 99-118. doi: 10.1177/0013164498058001009

Mendlowicz, M. V., \& Figueira, I. (2007). Transmissão intergeracional da violência familiar: $\mathrm{O}$ papel do transtorno de estresse pós-traumático. Rev. bras. psiquiatr, 29(1), 88-89. doi: 10.1590/ s1516-44462007000100023

Moraes, C. L., Hasselmann, M. H., \& Reichenheim, M. E. (2002). Portuguese transcultural adaptation of the Revised Conflict Tactics Scales (CTS2), an instrument used to identify partner violence. $\mathrm{Ca}$ dernos de Saúde Pública, 18, 163-175. doi: 10.1590/ S0102-311X2002000100017

Organização Mundial da Saúde, \& Krug, E. G. (2002). Relatório mundial sobre violência e saúde. Genebra: Organização Mundial da Saúde.

Ozório, C. D., Féres-Carneiro, T., \& Magalhães, A. S. (2017). Casamento dos pais e conjugalidade dos filhos: Do modelo tradicional ao contemporâneo. Pensando familias, 21(1), 20-32.

Panuzio, J., \& DiLillo, D. (2010). Physical, psychologi$\mathrm{cal}$, and sexual intimate partner aggression among Newlywed couples: Longitudinal prediction of marital satisfaction. Journal of Family Violence, 25, 689-699. doi: 10.1007/s10896-010-9328-2

Risman, A., Figueira, R. L., Vieira, G. M., \& de Azevedo, L. T. (2014). Abuso sexual intrafamiliar: Um olhar multifacetado para o incesto. América Latina, 26, 87105. Recuperado de http:/ / www.revistapsicolatina. org/wp-content/uploads/2014/07/5.-Abuso-sexual-intrafamiliar.pdf

Roberts, A., McLaughlin, K. A., Conron, K. J., \& Koenen, K. C. (2011). Adulthood stressors, history of childhood adversity, and risk of perpetration of intimate partner violence. American Journal of Preventive Medicine, 40(2), 128-138. doi: 10.1016/j. ypsy.2011.07.061

Rufatto, A. S. T. (2016). Crimes passionais: Direito e psicopatologia. Direito e Democracia, 12(1). Recuperado de http://www.periodicos.ulbra.br/index. $\mathrm{php} /$ direito/article/view/2608

Russo, G., Dantas, J., Nogueira, J., \& Trindade, H. (2014). Da omissão denunciada: Negligência intrafamiliar contra crianças e adolescentes no Creas/ Mossoró-RN. Revista SER Social, 16(34), 65-90. doi: 10.18315/argumentum.v6i1.5921

Sant'Anna, T. C., \& Penso, M. A. (2018). A transmissão geracional da violência na relação conjugal. Psicologia: teoria e pesquisa, 33(1).

Schraiber, L. B. et al., (2007). Prevalência da violência contra a mulher por parceiro íntimo em regiões do Brasil. Revista de Saúde Pública, 41(5), 797-807. doi: 10.1590/S0034-89102007000500014

Schuster, A. M. M., Alves, A. P., Correa, D. M., Bronzatti, G., Hornos, L., Rodrigues, R. P. \& Fernandes, T. G. (2009). Trangeracionalidade e psicoterapia de casal. Em I. L. C. Anton, O casal diante do espelho. São Paulo: Casa do Psicólogo.

Seleghim, M. R., \& Oliveira, M. L. F. (2013). Influência do ambiente familiar no consumo de crack em usuários. Acta Paul Enferm, 26(3), 263-268. doi: $10.1590 / \mathrm{s} 0103-21002013000300010$

Spencer, C., Cafferky, B., \& Stith, S. M. (2016). Gender differences in risk markers for perpetration of

Psico-USF, Bragança Paulista, v. 25, n. 3, p. 425-437, jul./set. 2020 
physical partner violence: Results from a meta-analytic review. Journal of family violence, 31(8), 981-984. doi: 10.1007/s10896-016-9860-9

Staples, J., Rellini, A. H., \& Roberts, S. P. (2012). Avoiding experiences: Sexual dysfunction in women with a history of sexual abuse in childhood and adolescence. Archives of Sexual Behavior, 41(2), 341-350. doi: 10.1007/s10508-011-9773-x

Straus, M. A. (2008). Dominance and symmetry in partner violence by male and female university in 32 nations. Children and Youth Services Review, 30, 252275. doi: 10.1016/j.childyouth.2007.10.004

Straus, M. A., Hamby, S. L., Buncy-McCoy, S., \& Sugarman, D. B. (1996). The revised Conflict Tactics Scale (CTS2): Development and preliminary psychometric data. Journal of Family Issues, 17(3), 283-316. doi: 10.1177/019251396017003001

Temple, J. R., Shorey, R. C., Tortolero, S. R., Wolfe, D. A., \& Stuart, G. L. (2013). Importance of gender and attitudes about violence in the relationship between exposure to interparental violence and the perpetration of teen dating violence. Child abuse \& neglect, 37(5), 343-352. doi: 10.1016/j. chiabu.2013.02.001

Timmons, A. C., Arbel, R., \& Margolin, G. (2017). Daily patterns of stress and conflict in couples: Associations with marital aggression and family-of-origin aggression. Journal of family psychology, 31(1), 93.

Turatti, M. C., \& Lucas, M. G. (2016). Compreendendo o ciúme na relação conjugal: Um olhar sistêmico. Unoesc \& Ciência-ACBS, 7(2), 145-152. Recuperado de https://editora.unoesc.edu.br/index.php/ acbs/article/view/11945/pdf

Turchik, J. A. (2012). Sexual victimization among male college students: Assault severity, sexual functioning, and health risk behaviors. Psychology of Men \& Masculinity, 13(3), 243-255. doi: 10.1037/a0024605

Vian, M., Mosmann, C. P., \& Falcke, D. (2019). Repercussões da conjugalidade em sintomas internalizantes e externalizantes em filhos adolescentes. Psicologia: Teoria e Pesquisa, 34, e34431.

Recebido em: 27/04/2018 Reformulado em: 13/05/2019

Aprovado em: 22/07/2019

Sobre as autoras:

Karla Rafaela Haack é psicóloga, doutora em Psicologia pela Universidade do Vale do Rio dos Sinos (Unisinos) e membro do Núcleo de Pesquisa de Família e Violência (NEFAV).

ORCID: https://orcid.org/0000-0001-8566-3847

E-mail:krh.psi@gmail.com

Denise Falcke é psicóloga, doutora em Psicologia, professora do Programa de Pós-Graduação em Psicologia da Universidade do Vale do Rio dos Sinos (Unisinos) e coordenadora do Núcleo de Pesquisa de Família e Violência (NEFAV).

ORCID: https://orcid.org/0000-0002-4653-1216

E-mail:dfalcke@unisinos.br

Contato com as autoras:

Universidade do Vale do Rio dos Sinos

Av. Unisinos, 950, Cristo Rei

São Leopoldo-RS, Brasil

CEP: 93022-000

Psico-USF, Bragança Paulista, v. 25, n. 3, p. 425-437, jul./set. 2020 